\title{
A major update of the International GLE Database: Correction for the variable GCR background
}

\author{
Ilya Usoskin, ${ }^{a, *}$ Sergey Koldobskiy, ${ }^{a, b}$ Gennady Kovaltsov, ${ }^{c}$ Agnieszka Gil, ${ }^{d}$ Inna \\ Usoskina, ${ }^{a}$ Teemu Willamo ${ }^{e}$ and Askar Ibragimov ${ }^{f}$ \\ ${ }^{a}$ University of Oulu, Finland. \\ ${ }^{b}$ National Research Nuclear University MEPhI, Moscow, Russia. \\ ${ }^{c}$ Ioffe Physical-Technical Institute, St. Petersburg, Russia. \\ ${ }^{d}$ Siedlce University, Institute of Mathematics, Poland. \\ ${ }^{e}$ University of Helsinki, Finland. \\ ${ }^{f}$ Independent researcher, Helsinki, Finland. \\ E-mail: ilya.usoskin@oulu.fi
}

\begin{abstract}
The main detector to provide data to study highly energetic (above $\approx 400 \mathrm{MeV}$ ) solar particles is the network of ground-based neutron monitors (NMs). Solar events recorded on the ground are called ground-level enhancements (GLEs). All GLE-related data from the NM network are collected in the International GLE Database (IGLED, https://gle.oulu.fi), which provides formal NM count-rate increases above the constant pre-increase level which is due to galactic cosmic rays (GCR). However, the basic formal assumption that the GCR background level remains constant throughout a GLE event is often violated. We have carefully revised the IGLED and provided a new data set of de-trended NM count-rate increases that accounts for the variable GCR background. This had led to a significant revision of the corresponding integral omnidirectional fluences of solar particles reconstructed from the GLE data. The database of the de-trended NM count rate is revised for most GLE events since 1956. Integral omnidirectional fluences were re-assessed for 58 GLE events and parameterized for 52 reasonably strong events by applying the modified Ellison-Ramaty spectral shape. This forms a basis for more precise studies of parameters of solar energetic particle events and thus for solar and space physics.
\end{abstract}

$37^{\text {th }}$ International Cosmic Ray Conference (ICRC 2021)

July 12 th - 23rd, 2021

Online - Berlin, Germany

\footnotetext{
${ }^{*}$ Presenter
} 


\section{Introduction}

While the slightly and slowly variable flux of galactic cosmic rays (GCRs) is always present in the Earth's radiation environment, sometimes sporadic solar eruptive events, such as flares or coronal mass ejections (CMEs), occur where ions can be accelerated to superthermal energies, forming intermittent fluxes of solar energetic particles (SEPs) [1]. Rarely, such SEPs can be accelerated to high energy (above $\approx 400 \mathrm{MeV}$ ) so that they can initiate a nucleonic-electromagnetic-muon cascade in the Earth's atmosphere, and secondaries of such cascades can reach the ground. These SEPs are detected by ground-based neutron monitors (NMs) as enhancements of their count rates and are called Ground-Level Enhancements (GLEs) [2]. At present, 72 GLEs have been registered since the 1940s, the first four were detected by the ionization chambers, and all GLEs since 1956 (GLE\#5) were detected by world NM network. The enhancements of the NM count rates, related to these events, are collected in the International GLE Database (IGLED - htpps://gle.oulu.fi). The IGLED is a result of a collective effort of the cosmic-ray community to systemize the data, and it is presently hosted by the University of Oulu. The IGLED provides verified information about the high-energy SEPs, thus complementing the lower-energy space-borne detectors, and forms a basis for numerous studies of solar eruptive events including reconstruction of energy spectra and angular distributions of SEPs during strong events (e.g., [3]).

Historically, the enhancement of a NM count rate associated to the arrival of SEPs to Earth was computed above the formal constant background pre-increase level caused by GCRs defined individually for each NM and each event as the averaged count-rate during two full hours before the onset of the GLE (slightly different definitions can be applied for earlier events). An example of this background is shown in Figure 1A, where the formal background is denoted by the blue dashed line. The corresponding enhancement is shown in Figure 1B as the blue curve. One can see that the duration and the integral intensity of the enhancement is ambiguous and depends on the time ascribed to the event, which sometimes cannot be decided from the NM data per se and requires additional information, e.g., from spacecraft. On the other hand, for some GLEs, the basic assumption of the constancy of the formal background level can be violated greatly distorting the GLE time profile and the integral strength. An example of that is seen in Figure 1A, when the GLE \#67 (02-Nov-2003) took place during the recovery phase of a Forbush decrease. An approximate evolution of the GCR-background level is shown by the red dashed curves interpolated between the pre- and post-event count rates. Similarly, the diurnal or other variability of the GCR background may distort the GLE signal. Accordingly, a revision of the IGLED dataset was needed in that the possible variability of the background level is taken into account.

Here we briefly present the result of such a revision and the corresponding update of the IGLED, with some details provided below. Full details of the methodology can be found elsewhere [4]. 

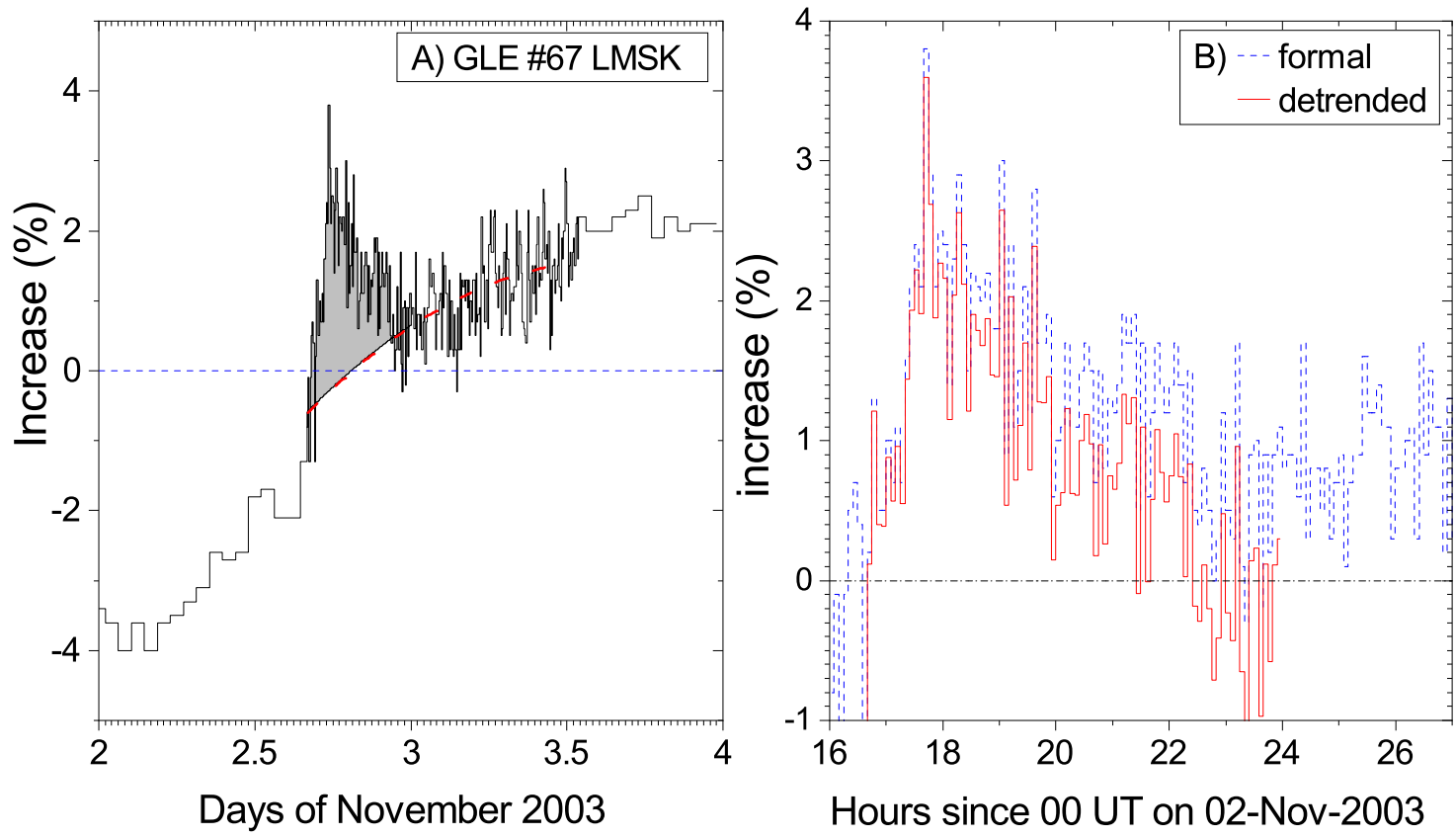

Figure 1: A) Count rate of LMSK (Lomnicky Stit) NM for GLE \#67 (02-Nov-2003). The blue-dashed horizontal line and red-dashed curve denote the 'formal' and 'realistic' baselines, respectively. The greyshaded area denotes the de-trended event integral strength. B) SEP-related excess of the NM count rate shown in panel A, after removal of the constant ('formal') and realistic variability ('detrended') baselines.

\section{Account for the variable background}

Traditionally, the GLE-related enhancement $I$ of the NM count rate was defined as the percentage increase above the constant background level $N_{0}$ as

$$
I_{\text {form }}=\frac{N(t)-N_{0}}{N_{0}} \cdot 100 \% .
$$

In order to account for the variable background, one needs to revise this definition as

$$
I_{\text {rev }}=\frac{N(t)-N_{\mathrm{b}}(t)}{N_{0}} \cdot 100 \%,
$$

where $N_{\mathrm{b}}(t)$ is the time-variable background, which was calculated using the following procedure applied to each individual NM record:

1. All the data were reduced to the hourly resolution, using the full UT hours.

2. Data points, corresponding to the GLE period were removed. The time period corresponding to GLE was defined either visually for high-response polar NMs or using the information from polar NMs in case of unclear signal in the lower-latitude NMs.

3. Two to eight hourly data-points from both sides of the GLE-related removed period were considered so that they define a smooth background level. 


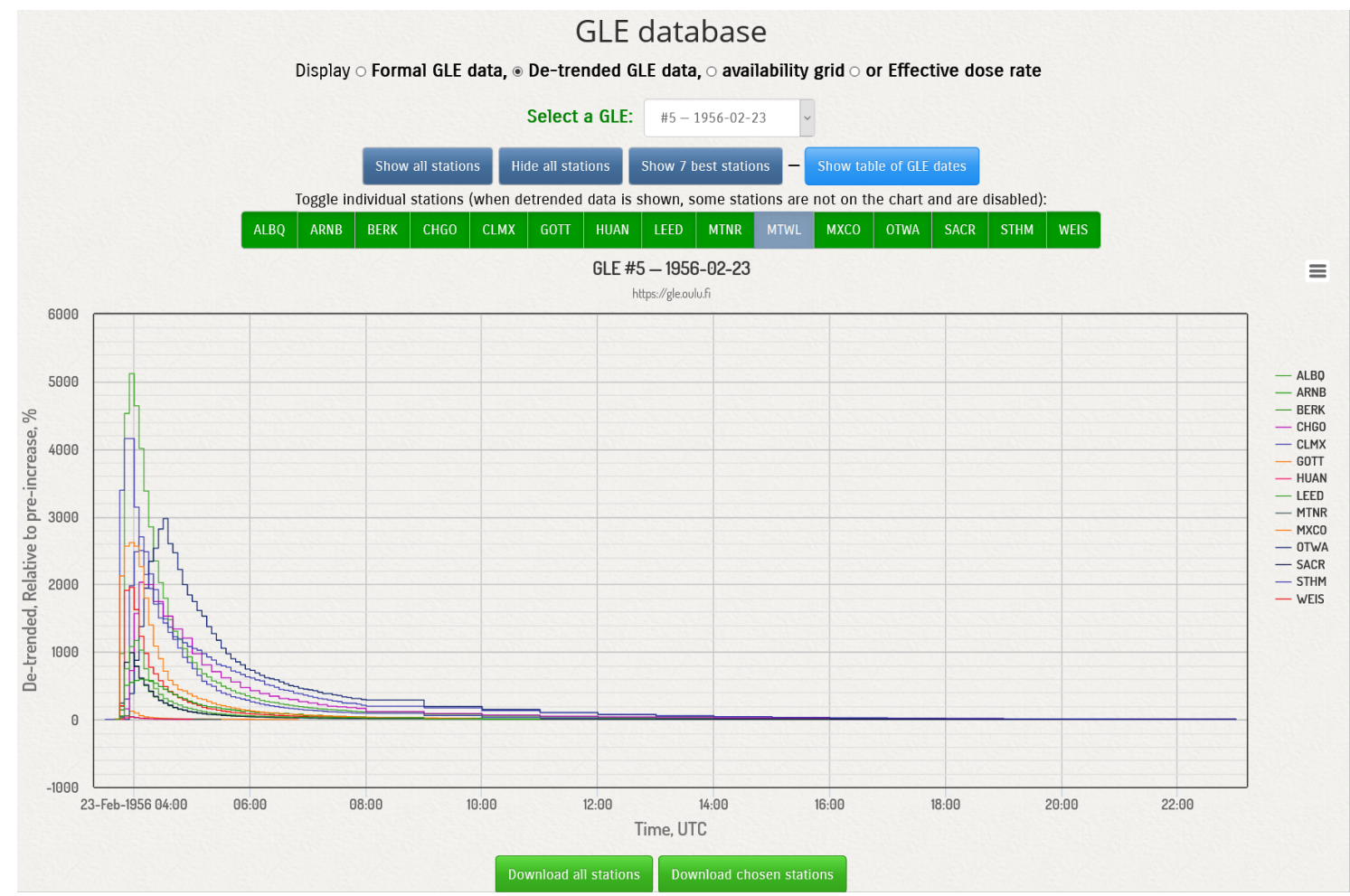

Figure 2: Screenshot of the IGLED (https://gle.oulu.fi). The "De-trended GLE data" radio-button is selected on the top. The drop-out menu "Select a GLE" provides a list of GLEs, individual NMs can be selected using the green buttons with acronyms. Time profiles of all the selected NM count-rate increases are plotted in the middle. The data can be downloaded using the green buttons in the bottom.

4. A parabolic fit was performed to the pre- and post-event data points. Interpolation of this fit to the GLE interval was considered as the background $N_{\mathrm{b}}$ above which the GLE response was calculated.

Then, using Equation 2, the percentage of enhancement was calculated, as exemplified in Figure 1B, where the red curve corresponds to $I_{\mathrm{rev}}$. One can see that the enhancement returns to the zero level towards the end of the event so that the integral event's strength does not depend on the exact definition of the event's duration.

Overall, 2824 individual records of NM count rates for 58 GLE events have been analyzed, summing to more than $2 \cdot 10^{5}$ count rate values. The values of relative enhancements $I_{\text {form }}$ and $I_{\text {rev }}$ are presented in the revised IGLED.

\section{Update of the IGLED}

The IGLED (https: / /gle . oulu . fi) has been updated in 2020 and includes now the revised enhancements, corrected for the variable background trend. A screenshot of the revised IGLED is shown in Figure 2 where a new radio-button De-trended GLE data has been added on the top. The default is the old option Formal GLE data, but the selection of the de-trended switches IGLED to the revised mode, where the increases corrected for the variable background level are depicted. 


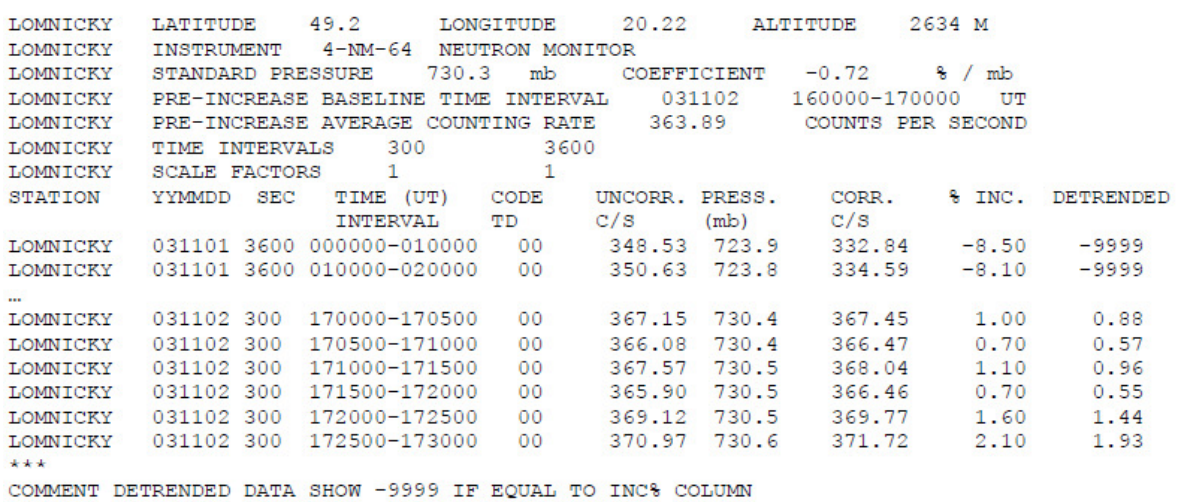

Figure 3: An example of a data file for the LMSK NM for GLE \#67 (see Figure 1) as downloaded from the IGLED (https://gle.oulu.fi). The file contains a header with metadata, the main part and a footer with possible comments. The two last columns in the main part list the formal $I_{\text {form }}$ and the revised de-trended $I_{\text {rev }}$ increase percentages, respectively.

Digital data, for selected NMs, can be downloaded as text files, an example of which is shown in Figure 3. Following the standard GLE format [5], data files contain a header with the necessary meta-data, the main part with time evolution of the uncorrected and pressure-corrected count rates, barometric pressure, as well as the increase data, followed by a footer with possible comments. After the revision, a new column called 'Detrended' was added to the data files. The sign '-9999' in this column implies that the detrended increase is the same as the formal increase.

\section{Estimates of the GLE energy spectra}

The revised data of detrended GLE responses of individual NMs made it possible to re-consider estimates of the total fluences (event-integrated spectra) of SEPs during the GLE events as well as their spectra. This work has been done in a series of papers $[4,6,7]$ using an updated NM yield function [8] and is only briefly mentioned here. We note that the revised fluences appear significantly (up to a factor of two) greater than previous estimates [9, 10]. The integral rigidity spectra can be well parameterized by the modified Ellison-Ramaty [11] shape:

$$
F(>R)=F_{0}\left(\frac{R}{1 \mathrm{GV}}\right)^{-\gamma} \exp \left(\frac{-R}{R_{0}}\right),
$$

where $R$ is the rigidity of particles and $R_{0}$ is the characteristic roll-off rigidity. The full table of the parameters for the analyzed GLEs is available elsewhere (Table 1 in [4]).

\section{Summary}

A full revision of the International GLE Database (IGLED htpps://gle . oulu . fi) has been performed with regard to verification and de-trending of the relative increases of count rates of NMs from the worlwide NM network for the known GLE events. In total, 2824 individual NM records for 58 GLE events have been re-analyzed and revised in the IGLED. The revision made it possible to revisit the estimates of the SEP integral spectra for 52 GLEs (except for very weak ones). This 
forms a solid basis for more reliable estimates of the SEP atmospheric effects such as radiation doses and ionization effects (e.g., [12, 13]).

\section{Acknowledgements}

This work was partly supported by the Academy of Finland (project 321882 ESPERA).

\section{References}

[1] R. Vainio, L. Desorgher, D. Heynderickx, M. Storini, E. Flückiger, R. B. Horne, G. A. Kovaltsov, K. Kudela, M. Laurenza, S. McKenna-Lawlor, H. Rothkaehl, and I. G. Usoskin, "Dynamics of the Earth's particle radiation environment," Space Sci. Rev., vol. 147, pp. 187231, 2009.

[2] S. Poluianov, I. Usoskin, A. Mishev, M. Shea, and D. Smart, "GLE and Sub-GLE Redefinition in the Light of High-Altitude Polar Neutron Monitors," Solar Phys., vol. 292, p. 176, 2017.

[3] A. Mishev and I. Usoskin, "Analysis of the Ground-Level Enhancements on 14 July 2000 and 13 December 2006 Using Neutron Monitor Data," Solar Phys., vol. 291, pp. 1225-1239, 2016.

[4] I. Usoskin, S. Koldobskiy, G. A. Kovaltsov, A. Gil, I. Usoskina, T. Willamo, and A. Ibragimov, "Revised GLE database: Fluences of solar energetic particles as measured by the neutronmonitor network since 1956," Astron. Astrophys., vol. 640, p. A17, 2020.

[5] I. Usoskin, A. Ibragimov, M. A. Shea, and D. F. Smart, "Database of Ground Level Enhancements (GLE) of High Energy Solar Proton Events," in 34th Intern. Cosmic Ray Conf. (ICRC2015), p. 54, 2015.

[6] S. A. Koldobskiy, G. A. Kovaltsov, A. L. Mishev, and I. G. Usoskin, "New Method of Assessment of the Integral Fluence of Solar Energetic ( $>1$ GV Rigidity) Particles from Neutron Monitor Data," Solar Phys., vol. 294, no. 7, p. 94, 2019.

[7] S. A. Koldobskiy, O. Raukunen, R. Vainio, G. Kovaltsov, and I. Usoskin, "New reconstruction of event-integrated spectra (spectral fluences) for major solar energetic particle events," Astron. Astrophys., vol. 647, p. A132, 2021.

[8] A. Mishev, S. Koldobskiy, G. Kovaltsov, A. Gil, and I. Usoskin, "Updated neutron-monitor yield function: Bridging between in-situ and ground-based cosmic ray measurements," $J$. Geophys. Res. (Space Phys.), vol. 125, p. e2019JA027433, 2020.

[9] A. Tylka and W. Dietrich, "A new and comprehensive analysis of proton spectra in groundlevel encahnced (gle) solar particle events," in 31th International Cosmic Ray Conference, (Lodź, Poland), p. icrc0273, Universal Academy Press, 2009.

[10] O. Raukunen, R. Vainio, A. J. Tylka, W. F. Dietrich, P. Jiggens, D. Heynderickx, M. Dierckxsens, N. Crosby, U. Ganse, and R. Siipola, "Two solar proton fluence models based on ground level enhancement observations," J. Space Weather Space Clim., vol. 8, no. 27, p. A04, 2018. 
[11] D. C. Ellison and R. Ramaty, "Shock acceleration of electrons and ions in solar flares," Astrophys. J., vol. 298, pp. 400-408, 1985.

[12] I. A. Mironova, K. L. Aplin, F. Arnold, G. A. Bazilevskaya, R. G. Harrison, A. A. Krivolutsky, K. A. Nicoll, E. V. Rozanov, E. Turunen, and I. G. Usoskin, "Energetic Particle Influence on the Earth's Atmosphere,” Space Sci Rev., vol. 194, pp. 1-96, 2015.

[13] A. Mishev, S. Tuohino, and I. Usoskin, "Neutron monitor count rate increase as a proxy for dose rate assessment at aviation altitudes during GLEs," J. Space Weather Space Clim., vol. 8, p. A46, 2018. 